\title{
Positive Solutions for a Fourth-Order Riemann-Stieltjes Integral Boundary Value Problem
}

\author{
Yujun Cui $\mathbb{D}^{1}{ }^{1}$ Donal O’Regan, ${ }^{2}$ and Jiafa $X u \mathbb{D}^{3}$ \\ ${ }^{1}$ State Key Laboratory of Mining Disaster Prevention and Control Co-Founded by Shandong Province \\ and the Ministry of Science and Technology, Shandong University of Science and Technology, Qingdao, \\ Shandong 266590, China \\ ${ }^{2}$ School of Mathematics, Statistics and Applied Mathematics, National University of Ireland, Galway, Ireland \\ ${ }^{3}$ School of Mathematical Sciences, Qufu Normal University, Qufu 273165, China
}

Correspondence should be addressed to Yujun Cui; cyj720201@163.com

Received 4 December 2018; Accepted 3 December 2019; Published 18 December 2019

Academic Editor: Higinio Ramos

Copyright ( 2019 Yujun Cui et al. This is an open access article distributed under the Creative Commons Attribution License, which permits unrestricted use, distribution, and reproduction in any medium, provided the original work is properly cited.

In this paper, we use the fixed point index to study the existence of positive solutions for the fourth-order Riemann-Stieltjes integral boundary value problem $\left\{\begin{array}{l}-x^{(4)}(t)=f\left(t, x(t), x^{\prime}(t), x^{\prime \prime}(t), x^{\prime \prime \prime}(t)\right), t \in(0,1) \\ x(0)=x^{\prime}(0)=x^{\prime \prime \prime}(1)=0, x^{\prime \prime}(0)=\alpha\left[x^{\prime \prime}(t)\right]\end{array}\right.$, where $f:[0,1] \times \mathbb{R}^{+} \times \mathbb{R}^{+} \times \mathbb{R}^{+} \times$ $\mathbb{R}^{+} \longrightarrow \mathbb{R}^{+}$is a continuous function and $\alpha\left[x^{\prime \prime}\right]$ denotes a linear function. Two existence theorems are obtained with some appropriate inequality conditions on the nonlinearity $f$, which involve the spectral radius of related linear operators. These conditions allow $f\left(t, z_{1}, z_{2}, z_{3}, z_{4}\right)$ to have superlinear or sublinear growth in $z_{i}, i=1,2,3,4$.

\section{Introduction}

In this paper, we investigate the existence of positive solutions for the following fourth-order Riemann-Stieltjes integral boundary value problem:

$$
\left\{\begin{array}{l}
-x^{(4)}(t)=f\left(t, x(t), x^{\prime}(t), x^{\prime \prime}(t), x^{\prime \prime \prime}(t)\right), \quad t \in(0,1), \\
x(0)=x^{\prime}(0)=x^{\prime \prime \prime}(1)=0, x^{\prime \prime}(0)=\alpha\left[x^{\prime \prime}(t)\right],
\end{array}\right.
$$

where $\alpha\left[x^{\prime \prime}(t)\right]=\int_{0}^{1} x^{\prime \prime}(t) \mathrm{d} \beta(t)$ denotes the RiemannStieltjes integral with a suitable function $\beta$ of bounded variation and

$$
(H 0) \alpha[1] \in[0,1) .
$$

The deformation of an elastic beam in an equilibrium state can be described by a fourth-order ordinary equation boundary value problem [1], and there are a large number of papers in the literature in this direction; for example, see [1-30] and the references therein. In [1], the author used Krasnosel'skii's fixed point theorem to establish one or two positive solutions for the fourth-order boundary value problem:

$$
\left\{\begin{array}{l}
u^{(4)}(t)+\beta u^{\prime \prime}(t)=\lambda f\left(t, u(t), u^{\prime \prime}(t)\right), \quad t \in(0,1) \\
u(0)=u(1)=\int_{0}^{1} p(s) u(s) \mathrm{d} s \\
u^{\prime \prime}(0)=u^{\prime \prime}(1)=\int_{0}^{1} q(s) u^{\prime \prime}(s) \mathrm{d} s
\end{array}\right.
$$


When $f \in C\left([0,1] \times \mathbb{R}^{+} \times \mathbb{R}^{-}, \mathbb{R}^{+}\right)$and in [2], the authors studied the existence of positive solutions for the fourth-order $m$-point boundary value problem:

$$
\left\{\begin{array}{l}
u^{(4)}+\alpha u^{\prime \prime}-\beta u=f(t, u), \quad t \in(0,1), \\
u(0)=\sum_{i=1}^{m-2} a_{i} u\left(\xi_{i}\right), u(1)=\sum_{i=1}^{m-2} b_{i} u\left(\xi_{i}\right) \\
u^{\prime \prime}(0)=\sum_{i=1}^{m-2} a_{i} u^{\prime \prime}\left(\xi_{i}\right), \\
u^{\prime \prime}(1)=\sum_{i=1}^{m-2} b_{i} u^{\prime \prime}\left(\xi_{i}\right)
\end{array}\right.
$$

where $f \in C\left([0,1] \times \mathbb{R}^{+}, \mathbb{R}^{+}\right)$satisfies superlinear and sublinear growth conditions:

$$
\begin{gathered}
\text { superlinear: } \lim \inf _{u \longrightarrow+\infty} \min _{t \in[0,1]} \frac{f(t, u)}{u}>\lambda^{*}, \\
\lim \sup _{u \longrightarrow 0^{+}} \max _{t \in[0,1]} \frac{f(t, u)}{u}<\lambda^{*}, \\
\text { sublinear: } \lim \inf _{u \longrightarrow 0^{+}} \min _{t \in[0,1]} \frac{f(t, u)}{u}>\lambda^{*}, \\
\lim \sup _{u \longrightarrow+\infty} \max _{t \in[0,1]} \frac{f(t, u)}{u}<\lambda^{*},
\end{gathered}
$$

where $\lambda^{*}$ is the first eigenvalue of the relevant linear operator.

In [3], the authors studied the existence of an iterative solution for the fourth-order boundary value problem:

$$
\left\{\begin{array}{l}
u^{(4)}(t)=f\left(t, u(t), u^{\prime}(t)\right), \quad t \in(0,1), \\
u(0)=u^{\prime}(0)=u^{\prime}(1)=u^{\prime \prime}(1)=0
\end{array}\right.
$$

where $f:[0,1] \times \mathbb{R}^{2} \longrightarrow \mathbb{R}$ is continuous and satisfies some appropriate Lipschitz condition, and in [4], the authors used the method of upper and lower solution to establish existence results for the fourth-order four-point boundary value problem on time scales:

$$
\left\{\begin{array}{l}
u^{\Delta \Delta \Delta \Delta}(t)=f\left(t, u(\sigma(t)), u^{\Delta \Delta}(t)\right), \quad t \in[0,1]_{\mathbb{T}} \\
u(0)=u\left(\sigma^{4}(1)\right)=0 \\
\alpha u^{\Delta \Delta}\left(\xi_{1}\right)-\beta u^{\Delta \Delta \Delta}\left(\xi_{1}\right)=0 \\
\gamma u^{\Delta \Delta}\left(\xi_{2}\right)+\eta u^{\Delta \Delta \Delta}\left(\xi_{2}\right)=0
\end{array}\right.
$$

where $f:[0,1] \times \mathbb{R} \times \mathbb{R} \longrightarrow \mathbb{R}$ is a continuous function.

There are only a few papers in the literature which consider general nonlinearities for fourth-order boundary value problems. The difficulty lies in a priori estimates for third-order derivatives, so some authors adopted a Nagumotype growth condition (see $(H 7)$ in Section 3 ) to overcome this difficulty; for example, see $[15,16,31-34]$ and the references therein. In [15], the author studied the existence of positive solutions for the fourth-order boundary value problem:

$$
\left\{\begin{array}{l}
u^{(4)}=f\left(t, u, u^{\prime}, u^{\prime \prime}, u^{\prime \prime \prime}\right), \quad t \in(0,1), \\
u(0)=u^{\prime}(0)=u^{\prime \prime}(1)=u^{\prime \prime \prime}(1)=0,
\end{array}\right.
$$

where $f \in C\left([0,1] \times \mathbb{R}^{+} \times \mathbb{R}^{+} \times \mathbb{R}^{+} \times \mathbb{R}^{-}, \mathbb{R}^{+}\right)$satisfies some inequality conditions, where $f$ grows both superlinearly and sublinearly about its variables $u, u^{\prime}, u^{\prime \prime}$, and $u^{\prime \prime \prime}$. When $f$ is superlinear, a Nagumo-type condition is used to restrict the growth of $f$ on $u^{\prime \prime}$ and $u^{\prime \prime \prime}$.

Integral boundary conditions arise in thermal conduction problems [35], semiconductor problems [36], and hydrodynamic problems [37], and there are some papers in the literature devoted to this direction (see $[1,9,19$, $34,38-48])$. In [19], the authors studied $p$-Laplacian fourthorder differential equations with Riemann-Stieltjes integral boundary conditions:

$$
\left\{\begin{array}{l}
\left(\phi_{p_{1}}\left(u^{\prime \prime}(t)\right)\right)^{\prime \prime}=\lambda^{p_{1}-1} a_{1}(t) f_{1}(t, u(t), v(t)), \quad 0<t<1, \\
\left(\phi_{p_{1}}\left(v^{\prime \prime}(t)\right)\right)^{\prime \prime}=\mu^{p_{2}-1} a_{2}(t) f_{2}(t, u(t), v(t)), \\
u(0)=u(1)=\int_{0}^{1} u(s) \mathrm{d} \xi_{1}(s), \\
\phi_{p_{1}}\left(u^{\prime \prime}(0)\right)=\phi_{p_{1}}\left(u^{\prime \prime}(1)\right)=\int_{0}^{1}\left(u^{\prime \prime}(s)\right) \mathrm{d} \eta_{1}(s) \phi_{p_{1}}, \\
v(0)=v(1)=\int_{0}^{1} v(s) \mathrm{d} \xi_{2}(s), \\
\phi_{p_{2}}\left(v^{\prime \prime}(0)\right)=\phi_{p_{2}}\left(v^{\prime \prime}(1)\right)=\int_{0}^{1} \phi_{p_{2}}\left(v^{\prime \prime}(s)\right) d \eta_{2}(s) .
\end{array}\right.
$$

The authors used fixed point theory in cones to obtain the existence of positive solutions for the above problem and provided the interval ranges of the parameters $\lambda$ and $\mu$ for these solutions.

In [38], the authors studied the fractional differential equation with a singular decreasing nonlinearity and a $p$ Laplacian operator:

$$
\left\{\begin{array}{l}
-D_{0+}^{\alpha}\left(\varphi_{p}\left(-D_{0+}^{\gamma} z\right)\right)(x)=f(x, z(x)), \quad 0<x<1, \\
z(0)=0, D_{0+}^{\gamma} z(0)=D_{0+}^{\gamma} z(1)=0, \\
z(1)=\int_{0}^{1} z(x) \mathrm{d} \chi(x) .
\end{array}\right.
$$

Using a double iterative technique, they showed that the above problem has a unique positive solution, and from an iterative technique, they established an appropriate sequence, which converges uniformly to the unique positive solution.

Motivated by the aforementioned works, the aim of this paper is to study the existence of positive solutions for the fourth-order Riemann-Stieltjes integral boundary value problem (1). The novelty is of two folds: (1) we provide some useful inequality conditions on $f$ involving the first eigenvalue of the relevant linear operator (these conditions imply that $f$ grows superlinearly and sublinearly) and (2) for the 
superlinear case, an appropriate Nagumo-type condition is used to restrict the growth of $f$ on $x^{\prime \prime \prime}$ in (1).

\section{Preliminaries}

In this section, we first transform (1) into an equivalent Hammerstein-type integral equation. For this, let $x^{\prime \prime}(t)=y(t)$, for $t \in[0,1]$. Then, from the conditions $x(0)=x^{\prime}(0)=0$, we have

$$
\begin{aligned}
& x^{\prime}(t)=\int_{0}^{t} x^{\prime \prime}(s) \mathrm{d} s=\int_{0}^{t} y(s) \mathrm{d} s, \\
& x(t)=\int_{0}^{t} x^{\prime}(s) \mathrm{d} s=\int_{0}^{t} \int_{0}^{s} y(\tau) \mathrm{d} \tau \mathrm{d} s .
\end{aligned}
$$

Therefore, substituting (11) into (1) gives

$$
\left\{\begin{array}{l}
-y^{\prime \prime}(t)=f\left(t, \int_{0}^{t} \int_{0}^{s} y(\tau) \mathrm{d} \tau \mathrm{d} s, \int_{0}^{t} y(s) \mathrm{d} s, y(t), y^{\prime}(t)\right), \quad t \in(0,1), \\
y(0)=\alpha[y(t)] \\
y^{\prime}(1)=0 .
\end{array}\right.
$$

Lemma 1. The problem (12) can be transformed into the Hammerstein-type integral equation:

$$
y(t)=\int_{0}^{1} G_{1}(t, s) f\left(s, \int_{0}^{s} \int_{0}^{\tau} y(r) \mathrm{d} r \mathrm{~d} \tau, \int_{0}^{s} y(\tau) d \tau, y(s), y^{\prime}(s)\right) \mathrm{d} s,
$$

where $G_{1}(t, s)=(1 / 1-\alpha[1]) \int_{0}^{1} K_{1}(t, s) d \beta(t)+K_{1}(t, s)$ and $K_{1}(t, s)=\min \{t, s\}$, for $t, s \in[0,1]$.

Proof. Using the function $g$ on $[0,1]$ to replace $f\left(t, \int_{0}^{t} \int_{0}^{s} y\right.$ $\left.(\tau) \mathrm{d} \tau \mathrm{d} s, \int_{0}^{t} y(s) \mathrm{d} s, y(t), y^{\prime}(t)\right)$ in (12), we consider the following problem:

$$
\left\{\begin{array}{l}
-y^{\prime \prime}(t)=g(t), \quad t \in(0,1), \\
y(0)=\alpha[y(t)], \\
y^{\prime}(1)=0 .
\end{array}\right.
$$

From the differential equation in (14), we obtain

$$
y(t)=\int_{0}^{t}(s-t) g(s) \mathrm{d} s+c_{1} t+c_{2}, \quad \text { for some } c_{i} \in \mathbb{R}, i=1,2,
$$

$$
\begin{aligned}
& y^{\prime}(t)=-\int_{0}^{t} g(s) \mathrm{d} s+c_{1}, \\
& y^{\prime \prime}(t)=-g(t) .
\end{aligned}
$$

The condition $y^{\prime}(1)=0$ implies that

$$
c_{1}=\int_{0}^{1} g(s) \mathrm{d} s
$$

Using the condition $y(0)=\alpha[y(t)]$, it enables us to obtain

$$
\begin{aligned}
c_{2} & =\int_{0}^{1}\left[\int_{0}^{t}(s-t) g(s) \mathrm{d} s+\int_{0}^{1} t g(s) \mathrm{d} s+c_{2}\right] \mathrm{d} \beta(t) \\
& =\int_{0}^{1}\left[\int_{0}^{1} K_{1}(t, s) g(s) \mathrm{d} s+c_{2}\right] \mathrm{d} \beta(t) .
\end{aligned}
$$

Hence, we have

$$
c_{2}=\frac{1}{1-\alpha[1]} \int_{0}^{1} \int_{0}^{1} K_{1}(t, s) g(s) \mathrm{d} s \mathrm{~d} \beta(t) .
$$

As a result, substituting $c_{1}$ and $c_{2}$ into (15) gives

and then

$$
\begin{aligned}
y(t) & =\int_{0}^{t}(s-t) g(s) \mathrm{d} s+\int_{0}^{1} t g(s) \mathrm{d} s+\frac{1}{1-\alpha[1]} \int_{0}^{1} \int_{0}^{1} K_{1}(t, s) g(s) \mathrm{d} s \mathrm{~d} \beta(t) \\
& =\int_{0}^{1} G_{1}(t, s) g(s) \mathrm{d} s .
\end{aligned}
$$

This completes the proof.

Let $E=C^{1}[0,1], y=\max \left\{\|y\|_{C},\left\|y^{\prime}\right\|_{C}\right\}$ with $\|y\|_{C}=$ $\max _{t \in[0,1]}|y(t)|$, and $P=\left\{y \in E: y(t) \geq 0, y^{\prime}(t) \geq 0, \forall t \in\right.$ $[0,1]\}$. Then, $(E,\|\cdot\|)$ is a Banach space, and $P$ is a cone on $E$. From Lemma 1, we can define an operator $A: P \longrightarrow P$ as follows:

$$
\begin{aligned}
(A y)(t)= & \int_{0}^{1} G_{1}(t, s) f\left(s, \int_{0}^{s} \int_{0}^{\tau} y(r) \mathrm{d} r \mathrm{~d} \tau,\right. \\
& \left.\int_{0}^{s} y(\tau) \mathrm{d} \tau, y(s), y^{\prime}(s)\right) \mathrm{d} s, \quad \text { for } y \in E .
\end{aligned}
$$

Then, $A$ is a completely continuous operator from the Arzelà-Ascoli theorem (this argument is standard). 


\section{Remark 1}

(i) In our work, we need the nonnegativity of Green's function $G_{1}$, so we have the following assumption:

$$
\text { (H1) } \int_{0}^{1} K_{1}(t, s) \mathrm{d} \beta(t) \geq 0, \quad \text { for } s \in[0,1] \text {. }
$$

(ii) We need some inequality conditions on the nonlinearity $f\left(t, z_{1}, z_{2}, z_{3}, z_{4}\right)$ with respect to the variables $z_{i}, i=1,2,3,4$. We consider some useful linear operators:

$$
\begin{aligned}
& \left(L_{1} y\right)(t)=\int_{0}^{1} G_{1}(t, s) \int_{0}^{s} \int_{0}^{\tau} y(r) \mathrm{d} r \mathrm{~d} \tau \mathrm{d} s:=\int_{0}^{1} G_{2}(t, s) y(s) \mathrm{d} s \\
& \left(L_{2} y\right)(t)=\int_{0}^{1} G_{1}(t, s) \int_{0}^{1} \int_{0}^{s} y(\tau) \mathrm{d} \tau \mathrm{d} s:=\int_{0}^{1} G_{3}(t, s) y(s) \mathrm{d} s, \quad \text { for } y \in E, t \in[0,1] .
\end{aligned}
$$

If we know the function $\beta$, we can obtain the functions $G_{2}$ and $G_{3}$.

Example 1. Let $\beta(t)=0$ for $t \in[0,1]$. Then, $G_{1}(t, s)=K_{1}(t, s)$, for $t, s \in[0,1]$. Let

$$
h(s, \tau)= \begin{cases}1, & 0 \leq \tau \leq s \leq 1 \\ 0, & 0 \leq s \leq \tau \leq 1\end{cases}
$$

and then from (22), we find

$$
G_{3}(t, s)=\int_{0}^{1} G_{1}(t, \tau) h(\tau, s) \mathrm{d} \tau, \quad \text { for } t, s \in[0,1] .
$$

We consider two cases:

(i) Case 1: when $0 \leq t \leq s \leq 1$, we have

$$
G_{3}(t, s)=\int_{0}^{t} 0 \cdot \tau \mathrm{d} \tau+\int_{t}^{s} 0 \cdot t \mathrm{~d} \tau+\int_{s}^{1} 1 \cdot t \mathrm{~d} \tau=t(1-s) .
$$

(ii) Case 2: when $0 \leq s \leq t \leq 1$, we have

$$
G_{3}(t, s)=\int_{0}^{s} 0 \cdot \tau \mathrm{d} \tau+\int_{s}^{t} 1 \cdot \tau \mathrm{d} \tau+\int_{t}^{1} 1 \cdot t \mathrm{~d} \tau=t-\frac{1}{2} t^{2}-\frac{1}{2} s^{2} .
$$

We now calculate $G_{2}$. For this, let $\int_{0}^{\tau} y(r) \mathrm{d} r=j(\tau)$ and $k(\tau, r)=\left\{\begin{array}{ll}0, & 0 \leq \tau \leq r \leq 1, \\ 1, & 0 \leq r \leq \tau \leq 1 .\end{array}\right.$ Then, we have

$$
\begin{aligned}
\int_{0}^{1} G_{1}(t, s) \int_{0}^{s} j(\tau) \mathrm{d} \tau \mathrm{d} s & =\int_{0}^{1} G_{3}(t, \tau) j(\tau) \mathrm{d} \tau \\
& =\int_{0}^{1} G_{3}(t, \tau) \int_{0}^{\tau} y(r) \mathrm{d} r \mathrm{~d} \tau \\
& =\int_{0}^{1} G_{3}(t, \tau) \int_{0}^{1} k(\tau, r) y(r) \mathrm{d} r \mathrm{~d} \tau .
\end{aligned}
$$

Therefore, from (22), we have

$G_{2}(t, s)=\int_{0}^{1} G_{3}(t, \tau) k(\tau, s) \mathrm{d} \tau, \quad$ for $t, s \in[0,1]$.

We consider two cases: (i) Case 1: when $0 \leq t \leq s \leq 1$, we have

$$
\begin{aligned}
G_{2}(t, s)= & \int_{0}^{t} 0 \cdot\left(t-\frac{1}{2} t^{2}-\frac{1}{2} \tau^{2}\right) \mathrm{d} \tau \\
& +\int_{t}^{s} 0 \cdot t(1-\tau) \mathrm{d} \tau+\int_{s}^{1} 1 \cdot t(1-\tau) \mathrm{d} \tau \\
= & t\left(\frac{1}{2}+\frac{1}{2} s^{2}-s\right) .
\end{aligned}
$$

(ii) Case 2: when $0 \leq s \leq t \leq 1$, we have

$$
\begin{aligned}
G_{2}(t, s)= & \int_{0}^{s} 0 \cdot\left(t-\frac{1}{2} t^{2}-\frac{1}{2} \tau^{2}\right) \mathrm{d} \tau \\
& +\int_{s}^{t} 1 \cdot\left(t-\frac{1}{2} t^{2}-\frac{1}{2} \tau^{2}\right) \mathrm{d} \tau+\int_{t}^{1} 1 \cdot t(1-\tau) \mathrm{d} \tau \\
= & \frac{1}{2} t-t s+\frac{1}{2} t^{2} s-\frac{1}{6} t^{3}+\frac{1}{6} s^{3}
\end{aligned}
$$

Example 2. Let $\beta(t)=(t / 2)$ for $t \in[0,1]$. Then, we have

$$
\begin{aligned}
\frac{1}{1-\alpha[1]} \int_{0}^{1} K_{1}(t, s) \mathrm{d} \beta(t)= & \int_{0}^{1} K_{1}(t, s) \mathrm{d} t=\int_{0}^{s} t \mathrm{~d} t \\
& +\int_{s}^{1} s \mathrm{~d} t=s-\frac{1}{2} s^{2}, \quad \text { for } s \in[0,1]
\end{aligned}
$$

Hence, $G_{1}(t, s)=s-(1 / 2) s^{2}+K_{1}(t, s)$, for $t, s \in[0,1]$. Note (22) and Example 1, so we only need to calculate

$$
\begin{aligned}
\int_{0}^{1}\left(\tau-\frac{1}{2} \tau^{2}\right) h(\tau, s) \mathrm{d} \tau & =\int_{s}^{1}\left(\tau-\frac{1}{2} \tau^{2}\right) \mathrm{d} \tau=\frac{1}{3}-\frac{1}{2} s^{2}+\frac{1}{6} s^{3}, \\
\int_{0}^{1}\left(\frac{1}{3}-\frac{1}{2} \tau^{2}+\frac{1}{6} \tau^{3}\right) k(\tau, s) \mathrm{d} \tau & =\int_{s}^{1}\left(\frac{1}{3}-\frac{1}{2} \tau^{2}+\frac{1}{6} \tau^{3}\right) \mathrm{d} \tau \\
& =\frac{5}{24}-\frac{1}{3} s+\frac{1}{6} s^{3}-\frac{1}{24} s^{4}, \quad \text { for } s \in[0,1] .
\end{aligned}
$$

Therefore, we obtain 


$$
\begin{aligned}
& G_{3}(t, s)=\frac{1}{3}-\frac{1}{2} s^{2}+\frac{1}{6} s^{3}+ \begin{cases}t(1-s), & 0 \leq t \leq s \leq 1, \\
t-\frac{1}{2} t^{2}-\frac{1}{2} s^{2}, & 0 \leq s \leq t \leq 1,\end{cases} \\
& G_{2}(t, s)=\frac{5}{24}-\frac{1}{3} s+\frac{1}{6} s^{3}-\frac{1}{24} s^{4}+ \begin{cases}t\left(\frac{1}{2}+\frac{1}{2} s^{2}-s\right), & 0 \leq t \leq s \leq 1, \\
\frac{1}{2} t-t s+\frac{1}{2} t^{2} s-\frac{1}{6} t^{3}+\frac{1}{6} s^{3}, & 0 \leq s \leq t \leq 1 .\end{cases}
\end{aligned}
$$

Lemma 2. (Krein-Rutman, see([49] theorem 19.3)). Let $P$ be a reproducing cone in a real Banach space $E$, and let $L: E \longrightarrow E$ be a compact linear operator with $L(P) \subset P$. If

$r(L)>0$, then there exists $\varphi \in P,\{0\}$ such that $L \varphi=r(L) \varphi$, where $r(L)$ is the spectral radius of $L$.

Lemma 3. For not all zero numbers $a, b, c$, and $d \geq 0$, we let

$$
\begin{aligned}
& \left(L_{a, b, c, d} y\right)(t)=a \int_{0}^{1} G_{2}(t, s) y(s) \mathrm{d} s+b \int_{0}^{1} G_{3}(t, s) y(s) \mathrm{d} s+c \int_{0}^{1} G_{1}(t, s) y(s) \mathrm{d} s+d \int_{0}^{1} G_{1}(t, s) y^{\prime}(s) \mathrm{d} s \\
& \left(L_{a, b, c} y\right)(t)=a \int_{0}^{1} G_{2}(t, s) y(s) \mathrm{d} s+b \int_{0}^{1} G_{3}(t, s) y(s) \mathrm{d} s+c \int_{0}^{1} G_{1}(t, s) y(s) \mathrm{d} s \\
& \left(L_{a, b, c}^{*} y\right)(s)=a \int_{0}^{1} G_{2}(t, s) y(t) \mathrm{d} t+b \int_{0}^{1} G_{3}(t, s) y(t) \mathrm{d} t+c \int_{0}^{1} G_{1}(t, s) y(t) \mathrm{d} t, \quad \text { for } y \in P .
\end{aligned}
$$

Then,

$$
\begin{aligned}
\left(\frac{1}{30} a+\frac{1}{8} b+\frac{1}{3} c+\frac{1}{3} d\right) \kappa_{1} & \leq r\left(L_{a, b, c, d}\right) \leq\left(\frac{1}{8} a+\frac{1}{3} b+\frac{1}{2} c+\frac{1}{2} d\right) \kappa_{2}, \\
\left(\frac{1}{30} a+\frac{1}{8} b+\frac{1}{3} c\right) \kappa_{1} & \leq r\left(L_{a, b, c}\right) \leq\left(\frac{1}{8} a+\frac{1}{3} b+\frac{1}{2} c\right) \kappa_{2}, \\
\left(\frac{1}{30} a+\frac{1}{8} b+\frac{1}{3} c\right) \kappa_{1} \leq r\left(L_{a, b, c}^{*}\right) & \leq\left(\frac{1}{8} a+\frac{1}{3} b+\frac{1}{2} c\right) \kappa_{2},
\end{aligned}
$$

Proof. We first give some inequalities for $G_{i}, i=1,2,3$. Note that $t s \leq K_{1}(t, s) \leq s$, for $t, s \in[0,1]$, and from the definition of $G_{1}$, we see that

$$
\begin{aligned}
\kappa_{1} t s & =\frac{\alpha[t]-\alpha[1]+1}{1-\alpha[1]} t s \leq G_{1}(t, s) \leq \frac{1}{1-\alpha[1]} s \\
& =\kappa_{2} s, \quad \text { for } t, s \in[0,1] .
\end{aligned}
$$

where $\kappa_{1}:=(\alpha[t]-\alpha[1]+1) /(1-\alpha[1])$ and $\kappa_{2}:=1 /(1-$ $\alpha[1])$.

$$
\begin{aligned}
& \frac{1}{2} \kappa_{1} t\left(1-s^{2}\right)= \int_{s}^{1} \kappa_{1} t \tau \mathrm{d} \tau \leq G_{3}(t, s)=\int_{0}^{1} G_{1}(t, \tau) h(\tau, s) \mathrm{d} \tau \leq \int_{s}^{1} \kappa_{2} \tau \mathrm{d} \tau=\frac{1}{2} \kappa_{2}\left(1-s^{2}\right) \\
& \quad \text { for } t, s \in[0,1], \\
& \frac{1}{6} \kappa_{1} t\left(2+s^{3}-3 s\right)= \int_{s}^{1} \frac{1}{2} \kappa_{1} t\left(1-\tau^{2}\right) \mathrm{d} \tau \leq G_{2}(t, s)=\int_{0}^{1} G_{3}(t, \tau) k(\tau, s) \mathrm{d} \tau \leq \int_{s}^{1} \frac{1}{2} \kappa_{2}\left(1-\tau^{2}\right) \mathrm{d} \tau, \\
&= \frac{1}{6} \kappa_{2}\left(2+s^{3}-3 s\right), \quad \text { for } t, s \in[0,1] .
\end{aligned}
$$


For convenience, let $\widetilde{\psi}_{0}(t)=t, \widetilde{\psi}_{1}(t)=(1 / 6)\left(2+t^{3}-\right.$ $3 t)$, and $\widetilde{\psi}_{2}(t)=(1 / 2)\left(1-t^{2}\right)$, for $t \in[0,1]$.

We only prove the inequalities in (35) about the spectral radius of $L_{a, b, c}$. For convenience, let $G_{a, b, c}(t, s)=a G_{2}(t, s)+$ $b G_{3}(t, s)+c G_{1}(t, s)$ for $t, s \in[0,1]$. Then, we have

$$
\left(L_{a, b, c} y\right)(t)=\int_{0}^{1} G_{a, b, c}(t, s) y(s) \mathrm{d} s, \quad \text { for } y \in P .
$$

Thus, we obtain

$$
\begin{aligned}
\left\|L_{a, b, c}\right\|= & \sup _{\|y\|=1}\left\|L_{a, b, c} y\right\|=\sup _{t \in[0,1],\|y\|=1} \int_{0}^{1} G_{a, b, c}(t, s) y(s) \mathrm{d} s \\
\leq & \max _{t \in[0,1]} \int_{0}^{1} G_{a, b, c}(t, s) \mathrm{d} s \leq \int_{0}^{1} \kappa_{2}\left[a \widetilde{\psi}_{1}(s)\right. \\
& \left.+b \widetilde{\psi}_{2}(s)+c \widetilde{\psi}_{0}(s)\right] \mathrm{d} s \\
\leq & \left(\frac{1}{8} a+\frac{1}{3} b+\frac{1}{2} c\right) \kappa_{2} .
\end{aligned}
$$

For all $n \in \mathbb{N}_{+}$, we note that

$$
\begin{aligned}
\left(L_{a, b, c}^{n} y\right)(t)= & L\left(L_{a, b, c}^{n-1} y\right)(t)=\int_{0}^{1} G_{a, b, c}\left(t, s_{n-1}\right)\left(L_{a, b, c}^{n-1} y\right)\left(s_{n-1}\right) \mathrm{d} s_{n-1} \\
= & \cdots \\
= & \underbrace{\int_{0}^{1} \int_{0}^{1} \cdots \int_{0}^{1} G_{a, b, c}\left(t, s_{n-1}\right) G_{a, b, c}\left(s_{n-1}, s_{n-2}\right)}_{n} \\
& \cdots G_{a, b, c}\left(s_{1}, s\right) y(s) \mathrm{d} s_{n-1} \mathrm{~d} s_{n-2} \cdots \mathrm{d} s, \quad \text { for } y \in P .
\end{aligned}
$$

Hence, we can obtain

$$
\begin{aligned}
& \left\|L_{a, b, c}^{n}\right\| \leq \max _{t \in[0,1]}^{\int_{n}^{1} \int_{0}^{1} \cdots \int_{0}^{1}} G_{a, b, c}\left(t, s_{n-1}\right) G_{a, b, c}\left(s_{n-1}, s_{n-2}\right) \\
& \cdots G_{a, b, c}\left(s_{1}, s\right) \mathrm{d} s_{n-1} \mathrm{~d} s_{n-2} \cdots \mathrm{d} s \\
& \leq \kappa_{2}^{n}\left[\int_{0}^{1}\left(a \widetilde{\psi}_{1}(s)+b \widetilde{\psi}_{2}(s)+c \widetilde{\psi}_{0}(s)\right) \mathrm{d} s\right]^{n} \\
& \leq\left(\frac{1}{8} a+\frac{1}{3} b+\frac{1}{2} c\right)^{n} \kappa_{2}^{n} .
\end{aligned}
$$

Gelfand's theorem implies that

$$
r\left(L_{a, b, c}\right)=\lim _{n \longrightarrow \infty} \sqrt[n]{\left\|L_{a, b, c}^{n}\right\|} \leq\left(\frac{1}{8} a+\frac{1}{3} b+\frac{1}{2} c\right) \kappa_{2} .
$$

Next, we introduce a conclusion in ([50], problem 2.1.4). Let $y \in C[0,1]$, and a functional $\mathrm{J}$ on $C[0,1]$ be as

$$
J(x)=\int_{0}^{1} x(t) y(t) \mathrm{d} t, \quad \forall x \in C[0,1] .
$$

Then, we have

$$
\|J\|=\int_{0}^{1}|y(t)| \mathrm{d} t .
$$

We note that there exists $t_{0} \in[0,1]$ such that $\max _{t \in[0,1]} \int_{0}^{1} G_{a, b, c}(t, s) \mathrm{d} s=\int_{0}^{1} G_{a, b, c}\left(t_{0}, s\right) \mathrm{d} s$. Then, in (38), for fixed $t$, we define a linear function:

$$
L_{a, b, c, t} y=\int_{0}^{1} G_{a, b, c}(t, s) y(s) \mathrm{d} s, \quad \text { for } y \in C[0,1],
$$

and thus

$$
\left\|L_{a, b, c, t}\right\|=\int_{0}^{1} G_{a, b, c}(t, s) \mathrm{d} s .
$$

Then, by the definition of the norm of linear function, we know that for all $\varepsilon>0$, there exists $y_{\varepsilon, t} \in C[0,1]$ with $\left\|y_{\varepsilon, t}\right\|=$ 1 such that

$$
\begin{aligned}
\int_{0}^{1} G_{a, b, c}(t, s) \mathrm{d} s & =\left\|L_{a, b, c, t}\right\|=\sup _{\|y\|=1}\left|L_{a, b, c, t} y\right| \geq\left|L_{a, b, c, t} y_{\varepsilon, t}\right| \\
& \geq \int_{0}^{1} G_{a, b, c}(t, s) \mathrm{d} s-\varepsilon .
\end{aligned}
$$

On the contrary, note from the definition of our norm we have

$$
\left\|L_{a, b, c} y\right\|=\sup _{t \in[0,1]}\left|L_{a, b, c, t} y\right| \geq\left|L_{a, b, c, t_{0}} y\right| .
$$

Consequently, we have

$$
\left\|L_{a, b, c}\right\|=\sup _{\|y\|=1}\left\|L_{a, b, c} y\right\| \geq\left\|L_{a, b, c, t_{0}} y_{\varepsilon, t_{0}}\right\| \geq \int_{0}^{1} G_{a, b, c}\left(t_{0}, s\right) \mathrm{d} s-\varepsilon .
$$

For the arbitrariness of $\varepsilon$, we have

$\left\|L_{a, b, c}\right\|=\int_{0}^{1} G_{a, b, c}\left(t_{0}, s\right) \mathrm{d} s=\max _{t \in[0,1]} \int_{0}^{1} G_{a, b, c}(t, s) \mathrm{d} s$.

Also, for all $n \in \mathbb{N}_{+}$, we obtain 


$$
\begin{aligned}
& \left\|L_{a, b, c}^{n}\right\|=\max _{t \in[0,1]} \underbrace{\int_{0}^{1} \int_{0}^{1} \cdots \int_{0}^{1}}_{n} G_{a, b, c}\left(t, s_{n-1}\right) G_{a, b, c}\left(s_{n-1}, s_{n-2}\right) \cdots G_{a, b, c}\left(s_{1}, s\right) \mathrm{d} s_{n-1} \mathrm{~d} s_{n-2} \cdots \mathrm{d} s \\
& \geq \kappa_{1}^{n}\left(\max _{t \in[0,1]} t\right) \underbrace{\int_{0}^{1} \int_{0}^{1} \cdots \int_{0}^{1}}_{n}\left[a \widetilde{\psi}_{1}\left(s_{n-1}\right)+b \widetilde{\psi}_{2}\left(s_{n-1}\right)+c \widetilde{\psi}_{0}\left(s_{n-1}\right)\right] \\
& \cdot s_{n-1}\left[a \widetilde{\psi}_{1}\left(s_{n-2}\right)+b \widetilde{\psi}_{2}\left(s_{n-2}\right)+c \widetilde{\psi}_{0}\left(s_{n-2}\right)\right] \cdots s_{1}\left[a \widetilde{\psi}_{1}(s)+b \widetilde{\psi}_{2}(s)+c \widetilde{\psi}_{0}(s)\right] \mathrm{d} s_{n-1} \mathrm{~d} s_{n-2} \cdots \mathrm{d} s \\
& =\kappa_{1}^{n} \int_{0}^{1}\left[a \widetilde{\psi}_{1}(s)+b \widetilde{\psi}_{2}(s)+c \widetilde{\psi}_{0}(s)\right] \mathrm{d} s\left(\int_{0}^{1}\left[a s \widetilde{\psi}_{1}(s)+b s \widetilde{\psi}_{2}(s)+c s \widetilde{\psi}_{0}(s)\right] \mathrm{d} s\right)^{n-1} \text {. }
\end{aligned}
$$

From Gelfand's theorem, we have

$$
\begin{aligned}
r\left(L_{a, b, c}\right) & =\lim _{n \longrightarrow \infty} \sqrt[n]{\left\|L_{a, b, c}^{n}\right\|} \geq \kappa_{1} \int_{0}^{1}\left[a s \widetilde{\psi}_{1}(s)+b s \tilde{\psi}_{2}(s)+c s \tilde{\psi}_{0}(s)\right] \mathrm{d} s \\
& \geq\left(\frac{1}{30} a+\frac{1}{8} b+\frac{1}{3} c\right) \kappa_{1} .
\end{aligned}
$$

This completes the proof.

Lemma 4. (see [51]). Let $E$ be a real Banach space and $P$ be a cone on E. Suppose that $\Omega \subset E$ is a bounded open set and that $A: \bar{\Omega} \cap P \longrightarrow P$ is a continuous compact operator. If there exists a $\omega_{0} \in P,\{0\}$ such that

$$
\omega-A \omega \neq \lambda \omega_{0}, \quad \forall \lambda \geq 0, \omega \in \partial \Omega \cap P,
$$

then $i(A, \Omega \cap P, P)=0$, where $i$ denotes the fixed point index on $P$.

Lemma 5. (see [51]). Let E be a real Banach space and $P$ be a cone on E. Suppose that $\Omega \subset E$ is a bounded open set with $0 \in \Omega$ and that $A: \bar{\Omega} \cap P \longrightarrow P$ is a continuous compact operator. If

$$
\omega-\lambda A \omega \neq 0, \quad \forall \lambda \in[0,1], \omega \in \partial \Omega \cap P,
$$

then $i(A, \Omega \cap P, P)=1$.

\section{Main Results}

In our paper, we let $B_{\rho}=\{y \in P:\|y\|<\rho\}$ for $\rho>0$. Now, $\partial B_{\rho}=\{y \in P:\|y\|=\rho\}$ and $\bar{B}_{\rho}=\{y \in P:\|y\| \leq \rho\}$. Now, we list our assumptions on the nonlinearity $f$.

$$
(\mathrm{H} 2) f \in C\left([0,1] \times \mathbb{R}^{+} \times \mathbb{R}^{+} \times \mathbb{R}^{+} \times \mathbb{R}^{+}, \mathbb{R}^{+}\right) \text {. }
$$

(H3) There exist not all zero numbers $a_{1}, b_{1}, c_{1}$, and $d_{1} \geq 0$ and $e_{1}>0$ such that $r\left(L_{a_{1}, b_{1}, c_{1}, d_{1}}\right)<1$, and $f\left(t, z_{1}, z_{2}, z_{3}, z_{4}\right) \leq a_{1} z_{1}+b_{1} z_{2}+c_{1} z_{3}+d_{1} z_{4}+e_{1}$, for $\left(t, z_{1}, z_{2}, z_{3}, z_{4}\right) \in[0,1] \times \mathbb{R}^{+} \times \mathbb{R}^{+} \times \mathbb{R}^{+} \times \mathbb{R}^{+}$.

(H4) There exist not all zero numbers $a_{2}, b_{2}, c_{2}$, and $d_{2} \geq 0$ and $\rho_{1}>0$ such that $r\left(L_{a_{2}, b_{2}, c_{2}, d_{2}}\right) \geq 1$, and $f\left(t, z_{1}, z_{2}, z_{3}, z_{4}\right) \geq a_{2} z_{1}+b_{2} z_{2}+c_{2} z_{3}+d_{2} z_{4}$, for $(t$, $\left.z_{1}, z_{2}, z_{3}, z_{4}\right) \in[0,1] \times\left[0, \rho_{1}\right] \times\left[0, \rho_{1}\right] \times\left[0, \rho_{1}\right] \times\left[0, \rho_{1}\right]$.

(H5) There exist not all zero numbers $a_{3}, b_{3}, c_{3}$, and $d_{3} \geq 0$ and $\rho_{2}>0$ such that $r\left(L_{a_{3}, b_{3}, c_{3}, d_{3}}\right)<1$, and $f\left(t, z_{1}, z_{2}, z_{3}, z_{4}\right) \leq a_{3} z_{1}+b_{3} z_{2}+c_{3} z_{3}+d_{3} z_{4}$, for $(t$, $\left.z_{1}, z_{2}, z_{3}, z_{4}\right) \in[0,1] \times\left[0, \rho_{2}\right] \times\left[0, \rho_{2}\right] \times\left[0, \rho_{2}\right] \times\left[0, \rho_{2}\right]$.

(H6) There exist not all zero numbers $a_{4}, b_{4}$, and $c_{4} \geq 0$ and $e_{2}>0$ such that $r\left(L_{a_{4}, b_{4}, c_{4}}^{*}\right)>1$, and $f\left(t, z_{1}, z_{2}\right.$, $\left.z_{3}, z_{4}\right) \geq a_{4} z_{1}+b_{4} z_{2}+c_{4} z_{3}-e_{2}$, for $\left(t, z_{1}, z_{2}, z_{3}, z_{4}\right) \in$ $[0,1] \times \mathbb{R}^{+} \times \mathbb{R}^{+} \times \mathbb{R}^{+} \times \mathbb{R}^{+}$.

(H7) For any $M>0$, there exists a positive continuous function $H_{M}(\varrho)$ on $\mathbb{R}^{+}$such that $\int_{0}^{+\infty}\left(\varrho \mathrm{d} \varrho / H_{M}(\varrho)+\delta_{0}\right)=$ $+\infty, \forall \delta_{0}>0$, and $f\left(t, z_{1}, z_{2}, z_{3}, z_{4}\right) \leq H_{M}\left(z_{4}\right)$, for $\left(t, z_{1}, z_{2}\right.$, $\left.z_{3}, z_{4}\right) \in[0,1] \times[0, M] \times[0, M] \times[0, M] \times \mathbb{R}^{+}$.

Remark 2. Considering Lemma 3, one can adjust the coefficients $a_{i}, b_{i}, c_{i}$, and $d_{i}(i=1,2,3,4)$ such that the spectral radii $r\left(L_{a_{i}, b_{i}, c_{i}, d_{i}}\right), r\left(L_{a_{4}, b_{4}, c_{4}}\right), r\left(L_{a_{4}, b_{4}, c_{4}}^{*}\right)(i=1,2,3)$ satisfy their respective conditions in $(\mathrm{H} 3)-(\mathrm{H} 6)$.

Theorem 1. Suppose that (HO-H4) hold. Then, (1) has at least one positive solution.

Proof. Let $W=\{y \in P: y=\lambda A y, \lambda \in[0,1]\}$. Now, we prove that $\mathrm{W}$ is a bounded set in $P$. If $y \in W$, then from (H3), we have

$$
\begin{aligned}
y(t) & =\lambda(A y)(t) \leq(A y)(t) \leq \int_{0}^{1} G_{1}(t, s)\left(a_{1} \int_{0}^{s} \int_{0}^{\tau} y(r) \mathrm{d} r \mathrm{~d} \tau+b_{1} \int_{0}^{s} y(\tau) \mathrm{d} \tau+c_{1} y(s)+d_{1} y^{\prime}(s)+e_{1}\right) \mathrm{d} s \\
& =\int_{0}^{1}\left(a_{1} G_{2}(t, s)+b_{1} G_{3}(t, s)+c_{1} G_{1}(t, s)\right) y(s) \mathrm{d} s+d_{1} \int_{0}^{1} G_{1}(t, s) y^{\prime}(s) \mathrm{d} s+e_{1} \eta(t) \\
& =\left(L_{a_{1}, b_{1}, c_{1}, d_{1}} y\right)(t)+e_{1} \eta(t),
\end{aligned}
$$


where

$$
\begin{aligned}
\eta(t) & =\int_{0}^{1}\left[\frac{1}{1-\alpha[1]} \int_{0}^{1} K_{1}(t, s) \mathrm{d} \beta(t)+K_{1}(t, s)\right] \mathrm{d} s \\
& =\frac{1}{1-\alpha[1]} \int_{0}^{1} \int_{0}^{1} K_{1}(t, s) \mathrm{d} \beta(t) \mathrm{d} s+t-\frac{1}{2} t^{2},
\end{aligned}
$$

for $t \in[0,1]$. This implies that

$$
\left(\left(I-L_{a_{1}, b_{1}, c_{1}, d_{1}}\right) y\right)(t) \leq e_{1} \eta(t) .
$$

Note that $\eta \in P\left(\eta^{\prime}(t)=1-t \geq 0, \forall t \in[0,1]\right), y \in W$, and we obtain

$$
\begin{aligned}
y^{\prime}(t) & =\lambda(A y)^{\prime}(t)=\lambda \int_{t}^{1} f\left(s, \int_{0}^{s} \int_{0}^{\tau} y(r) \mathrm{d} r \mathrm{~d} \tau, \int_{0}^{s} y(\tau) d \tau, y(s), y^{\prime}(s)\right) \mathrm{d} s \\
& \leq \int_{0}^{1} \frac{\partial G_{1}}{\partial t}(t, s)\left(a_{1} \int_{0}^{s} \int_{0}^{\tau} y(r) \mathrm{d} r \mathrm{~d} \tau+b_{1} \int_{0}^{s} y(\tau) d \tau+c_{1} y(s)+d_{1} y^{\prime}(s)+e_{1}\right) \mathrm{d} s \\
& =\int_{0}^{1} \frac{\partial}{\partial t}\left(a_{1} G_{2}(t, s)+b_{1} G_{3}(t, s)+c_{1} G_{1}(t, s)\right) y(s) \mathrm{d} s+d_{1} \int_{0}^{1} \frac{\partial}{\partial t} G_{1}(t, s) y^{\prime}(s) \mathrm{d} s+e_{1} \eta^{\prime}(t) \\
& =\left(L_{a_{1}, b_{1}, c_{1}, d_{1}} y\right)^{\prime}(t)+e_{1} \eta^{\prime}(t) .
\end{aligned}
$$

Thus,

$$
\left(\left(I-L_{a_{1}, b_{1}, c_{1}, d_{1}}\right) y\right)^{\prime}(t) \leq e_{1} \eta^{\prime}(t)
$$

Since $r\left(L_{a_{1}, b_{1}, c_{1}, d_{1}}\right)<1$, we know that $I-L_{a_{1}, b_{1}, c_{1}, d_{1}}$ has a bounded inverse operator $\left(I-L_{a_{1}, b_{1}, c_{1}, d_{1}}\right)^{-1}$, with

$$
\begin{aligned}
\left(I-L_{a_{1}, b_{1}, c_{1}, d_{1}}\right)^{-1}= & I+L_{a_{1}, b_{1}, c_{1}, d_{1}}+L_{a_{1}, b_{1}, c_{1}, d_{1}}^{2}+\cdots \\
& +L_{a_{1}, b_{1}, c_{1}, d_{1}}^{n}+\cdots
\end{aligned}
$$

Note that $L_{a_{1}, b_{1}, c_{1}, d_{1}}(P) \subset P$, and we obtain (I$\left.L_{a_{1}, b_{1}, c_{1}, d_{1}}\right)^{-1}(P) \subset P$. Therefore,

$$
\begin{array}{r}
y(t) \leq\left(\left(I-L_{a_{1}, b_{1}, c_{1}, d_{1}}\right)^{-1} e_{1} \eta\right)(t), \\
y^{\prime}(t) \leq\left(\left(I-L_{a_{1}, b_{1}, c_{1}, d_{1}}\right)^{-1} e_{1} \eta\right)^{\prime}(t), \\
\text { for } t \in[0,1] .
\end{array}
$$

This implies that

$$
\begin{aligned}
\|y\|_{C} & \leq\left\|\left(\left(I-L_{a_{1}, b_{1}, c_{1}, d_{1}}\right)^{-1} e_{1} \eta\right)\right\|_{C}, \\
\left\|y^{\prime}\right\|_{C} & \leq\left\|\left(\left(I-L_{a_{1}, b_{1}, c_{1}, d_{1}}\right)^{-1} e_{1} \eta\right)^{\prime}\right\|_{C} .
\end{aligned}
$$

Therefore, we have

$$
\|y\| \leq\left\|\left(\left(I-L_{a_{1}, b_{1}, c_{1}, d_{1}}\right)^{-1} e_{1} \eta\right)\right\| .
$$

That is, $W$ is bounded. Now, we can select $R_{1}>\max \left\{\sup W, \rho_{1}\right\}\left(\rho_{1}\right.$ is defined in $\left.(\mathrm{H} 4)\right)$ such that

$$
y \neq \lambda A y, \quad \text { for } y \in \partial B_{R_{1}} \cap P \text { and } \lambda \in[0,1] \text {. }
$$

From Lemma 5, we have

$$
i\left(A, B_{R_{1}} \cap P, P\right)=1 .
$$

On the contrary, since $L_{a_{2}, b_{2}, c_{2}, d_{2}}(P) \subset P$ and $r\left(L_{a_{2}, b_{2}, c_{2}, d_{2}}\right) \geq 1$, it follows from Lemma 2 that there exists $\varphi_{0} \in P \mid\{0\} \quad$ such that $L_{a_{2}, b_{2}, c_{2}, d_{2}} \varphi_{0}=r\left(L_{a_{2}, b_{2}, c_{2}, d_{2}}\right) \varphi_{0}$ and $\varphi_{0}=r\left(L_{a_{2}, b_{2}, c_{2}, d_{2}}\right)^{-1} L_{a_{2}, b_{2}, c_{2}, d_{2}} \varphi_{0} \in P$. Now, we show that

$$
y-A y \neq \lambda \varphi_{0}, \quad \text { for } y \in \partial B_{\rho_{1}} \cap P, \lambda \geq 0 .
$$

If this claim is false, then there exist $y_{0} \in \partial B_{\rho_{1}} \cap P$ and $\lambda_{0} \geq 0$ such that $y_{0}-A y_{0}=\lambda_{0} \varphi_{0}$. Note that $\lambda_{0}>0$ (otherwise, the theorem is proved). Then, from (H4), we have

$$
\begin{aligned}
\left(A y_{0}\right)(t) & =\int_{0}^{1} G_{1}(t, s) f\left(s, \int_{0}^{s} \int_{0}^{\tau} y_{0}(r) \mathrm{d} r \mathrm{~d} \tau, \int_{0}^{s} y_{0}(\tau) \mathrm{d} \tau, y_{0}(s), y_{0}^{\prime}(s)\right) \mathrm{d} s \\
& \geq \int_{0}^{1} G_{1}(t, s)\left(a_{2} \int_{0}^{s} \int_{0}^{\tau} y_{0}(r) \mathrm{d} r \mathrm{~d} \tau+b_{2} \int_{0}^{s} y_{0}(\tau) d \tau+c_{2} y_{0}(s)+d_{2} y_{0}^{\prime}(s)\right) \mathrm{d} s \\
& =\int_{0}^{1}\left(a_{2} G_{2}(t, s)+b_{2} G_{3}(t, s)+c_{2} G_{1}(t, s)\right) y_{0}(s) \mathrm{d} s+d_{2} \int_{0}^{1} G_{1}(t, s) y_{0}^{\prime}(s) \mathrm{d} s \\
& =\left(L_{a_{2}, b_{2}, c_{2}, d_{2}} y_{0}\right)(t)
\end{aligned}
$$


which implies that

$$
y_{0}=A y_{0}+\lambda_{0} \varphi_{0} \geq L_{a_{2}, b_{2}, c_{2}, d_{2}} y_{0}+\lambda_{0} \varphi_{0} \geq \lambda_{0} \varphi_{0} .
$$

Let $\lambda^{*}=\sup \left\{\lambda>0: y_{0} \geq \lambda \varphi_{0}\right\}$. Then, $\lambda_{0} \in\left\{\lambda>0: y_{0} \geq\right.$ $\left.\lambda \varphi_{0}\right\}$ and $y_{0} \geq \lambda^{*} \varphi_{0}$. However, we note that $y_{0} \geq L_{a_{2}, b_{2}, c_{2}, d_{2}} y_{0}+$ $\lambda_{0} \varphi_{0} \geq L_{a_{2}, b_{2}, c_{2}, d_{2}} \lambda^{*} \varphi_{0}+\lambda_{0} \varphi_{0}=\left(\lambda^{*} r\left(L_{a_{2}, b_{2}, c_{2}, d}\right)+\lambda_{0}\right) \varphi_{0}$, and this contradicts the definition of $\lambda^{*}$ for $r\left(L_{a_{2}, b_{2}, c_{2}, d_{2}}\right) \geq 1$. Therefore, (66) holds, as required. From Lemma 4, we have

$$
i\left(A, B_{\rho_{1}} \cap P, P\right)=0 .
$$

From (65) and (69), we have

$$
i\left(A,\left(B_{R_{1}} \backslash \bar{B}_{\rho_{1}}\right) \cap P, P\right)=i\left(A, B_{R_{1}} \cap P, P\right)-i\left(A, B_{\rho_{1}} \cap P, P\right)=1,
$$

and hence $A$ has at least one fixed point in $\left(B_{R_{1}}, \bar{B}_{\rho_{1}}\right) \cap P$, i.e., (1) has at least one positive solution. This completes the proof.

Theorem 2. Suppose that (HO-H2) and (H5-H7) hold. Then, (1) has at least one positive solution.

Proof. We show that

$$
y \neq \lambda A y, \quad \text { for } y \in \partial B_{\rho_{2}} \cap P, \lambda \in[0,1] .
$$

If the claim is false, then there exist $y_{1} \in \partial B_{\rho_{2}} \cap P$ and $\lambda_{1} \in[0,1]$ such that $y_{1}(t)=\lambda_{1}\left(A y_{1}\right)(t)$, for $t \in[0,1]$. For $t \in[0,1]$, from (H5) we have

$$
\begin{aligned}
y_{1}(t) & \leq \int_{0}^{1} G_{1}(t, s) f\left(s, \int_{0}^{s} \int_{0}^{\tau} y_{1}(r) \mathrm{d} r \mathrm{~d} \tau, \int_{0}^{s} y_{1}(\tau) \mathrm{d} \tau, y_{1}(s), y_{1}^{\prime}(s)\right) \mathrm{d} s \\
& \leq \int_{0}^{1} G_{1}(t, s)\left(a_{3} \int_{0}^{s} \int_{0}^{\tau} y_{1}(r) \mathrm{d} r \mathrm{~d} \tau+b_{3} \int_{0}^{s} y_{1}(\tau) \mathrm{d} \tau+c_{3} y_{1}(s)+d_{3} y_{1}^{\prime}(s)\right) \mathrm{d} s \\
& =\left(L_{a_{3}, b_{3}, c_{3}, d_{3}} y_{1}\right)(t) .
\end{aligned}
$$

Also, $y_{1}^{\prime}(t)=\lambda_{1}\left(A y_{1}\right)^{\prime}(t)$, for $t \in[0,1]$ implies that

$$
\begin{aligned}
y_{1}^{\prime}(t) & =\lambda \int_{t}^{1} f\left(s, \int_{0}^{s} \int_{0}^{\tau} y_{1}(r) \mathrm{d} r \mathrm{~d} \tau, \int_{0}^{s} y_{1}(\tau) \mathrm{d} \tau, y_{1}(s), y_{1}^{\prime}(s)\right) \mathrm{d} s \\
& \leq \int_{0}^{1} \frac{\partial G_{1}}{\partial t}(t, s) f\left(s, \int_{0}^{s} \int_{0}^{\tau} y_{1}(r) \mathrm{d} r \mathrm{~d} \tau, \int_{0}^{s} y_{1}(\tau) \mathrm{d} \tau, y_{1}(s), y_{1}^{\prime}(s)\right) \mathrm{d} s \\
& \leq \int_{0}^{1} \frac{\partial G_{1}}{\partial t}(t, s)\left(a_{3} \int_{0}^{s} \int_{0}^{\tau} y_{1}(r) \mathrm{d} r \mathrm{~d} \tau+b_{3} \int_{0}^{s} y_{1}(\tau) \mathrm{d} \tau+c_{3} y_{1}(s)+d_{3} y_{1}^{\prime}(s)\right) \mathrm{d} s \\
& =\left(L_{a_{3}, b_{3}, c_{3}, d_{3}} y_{1}\right)^{\prime}(t) .
\end{aligned}
$$
have

Note that $L_{a_{3}, b_{3}, c_{3}, d_{3}}(P) \subset P$ and $r\left(L_{a_{3}, b_{3}, c_{3}, d_{3}}\right)<1$, and we

$$
\begin{gathered}
\left(\left(I-L_{a_{3}, b_{3}, c_{3}, d_{3}}\right) y_{1}\right)(t) \leq 0, \\
\left(\left(I-L_{a_{3}, b_{3}, c_{3}, d_{3}}\right) y_{1}\right)^{\prime}(t) \leq 0, \\
\text { for } t \in[0,1] .
\end{gathered}
$$

Therefore,

$$
\begin{aligned}
y_{1}(t)= & 0, \\
y^{\prime}(t)=0, & \\
& \text { for } t \in[0,1] .
\end{aligned}
$$

This contradicts the fact that $y_{1} \in \partial B_{\rho_{2}} \cap P$. Hence, (71) is true, as required. From Lemma 5, we have

$$
i\left(A, B_{\rho_{2}} \cap P, P\right)=1 \text {. }
$$

On the contrary, from Lemma 2 there exists $\psi_{1} \in P \mid\{0\}$ such that $\left(L_{a_{4}, b_{4}, c_{4}}^{*} \psi_{1}\right)(s)=r\left(L_{a_{4}, b_{4}, c_{4}}^{*}\right) \psi_{1}(s)$, for $s \in[0,1]$. Let $U=\left\{y \in P: y-A y=\lambda \varphi_{2}\right.$, for $\left.\lambda \geq 0\right\}$, where $\varphi_{2}(t)=t-$ $(1 / 2) t^{2} \in P$, for $t \in[0,1]$. Note that $\lambda>0$ (otherwise, the theorem is proved). We shall show that $U$ is a bounded set in $P$. If $y \in P$, then from (H6), we have 


$$
\begin{aligned}
y(t) & =(A y)(t)+\lambda \varphi_{2}(t) \geq(A y)(t) \\
& \geq \int_{0}^{1} G_{1}(t, s)\left(a_{4} \int_{0}^{s} \int_{0}^{\tau} y(r) \mathrm{d} r \mathrm{~d} \tau+b_{4} \int_{0}^{s} y(\tau) \mathrm{d} \tau+c_{4} y(s)-e_{2}\right) \mathrm{d} s \\
& \geq \int_{0}^{1}\left(a_{4} G_{2}(t, s)+b_{4} G_{3}(t, s)+c_{4} G_{1}(t, s)\right) y(s) \mathrm{d} s-e_{2} \int_{0}^{1} G_{1}(t, s) \mathrm{d} s \\
& \geq \int_{0}^{1}\left(a_{4} G_{2}(t, s)+b_{4} G_{3}(t, s)+c_{4} G_{1}(t, s)\right) y(s) \mathrm{d} s-\frac{1}{2} \kappa_{2} e_{2} .
\end{aligned}
$$

Multiplying both sides of the above inequality by $\psi_{1}(t)$ and integrating from 0 to 1 yields

$$
\begin{aligned}
\int_{0}^{1} y(t) \psi_{1}(t) \mathrm{d} t \geq & \int_{0}^{1} \psi_{1}(t) \int_{0}^{1}\left(a_{4} G_{2}(t, s)+b_{4} G_{3}(t, s)\right. \\
& \left.+c_{4} G_{1}(t, s)\right) y(s) \mathrm{d} s \mathrm{~d} t-\frac{1}{2} \kappa_{2} e_{2} \int_{0}^{1} \psi_{1}(t) \mathrm{d} t \\
\geq & r\left(L_{a_{4}, b_{4}, c_{4}}^{*}\right) \int_{0}^{1} y(t) \psi_{1}(t) \mathrm{d} t \\
& -\frac{1}{2} \kappa_{2} e_{2} \int_{0}^{1} \psi_{1}(t) \mathrm{d} t .
\end{aligned}
$$

This, together with $r\left(L_{a_{4}, b_{4}, c_{4}}^{*}\right)>1$, implies that

$$
\int_{0}^{1} y(t) \psi_{1}(t) \mathrm{d} t \leq \frac{\kappa_{2} e_{2} \int_{0}^{1} \psi_{1}(t) \mathrm{d} t}{2 r\left(L_{a_{4}, b_{4}, c_{4}}^{*}\right)-2}
$$

Note that $y \in U$, and we have

$$
\begin{aligned}
& y^{\prime}(t)=(A y)^{\prime}(t)+\lambda \varphi_{2}^{\prime}(t)=\int_{t}^{1} f\left(s, \int_{0}^{s} \int_{0}^{\tau} y(r) \mathrm{d} r \mathrm{~d} \tau, \int_{0}^{s} y(\tau) \mathrm{d} \tau, y(s), y^{\prime}(s)\right) \mathrm{d} s+\lambda(1-t) \geq 0, \\
& y^{\prime \prime}(t)=(A y)^{\prime \prime}(t)+\lambda \varphi_{2}^{\prime \prime}(t)=-f\left(t, \int_{0}^{t} \int_{0}^{\tau} y(r) \mathrm{d} r \mathrm{~d} \tau, \int_{0}^{t} y(\tau) \mathrm{d} \tau, y(t), y^{\prime}(t)\right)-\lambda \leq 0 .
\end{aligned}
$$
Hence,

Then, $y$ is a concave and increasing function on $[0,1]$.

$$
\begin{aligned}
\int_{0}^{1} y(t) \psi_{1}(t) \mathrm{d} t & =\int_{0}^{1} y\left(\frac{t}{1} \cdot 1+\frac{1-t}{1} \cdot 0\right) \psi_{1}(t) \mathrm{d} t \\
& \geq \int_{0}^{1}(t y(1)+(1-t) y(0)) \psi_{1}(t) \mathrm{d} t \\
& \geq \int_{0}^{1} t y(1) \psi_{1}(t) \mathrm{d} t .
\end{aligned}
$$

This enables us to obtain

$$
\|y\|_{C}=y(1) \leq \frac{\kappa_{2} e_{2} \int_{0}^{1} \psi_{1}(t) \mathrm{d} t}{2 r\left(L_{a_{4}, b_{4}, c_{4}}^{*}\right)-2}\left[\int_{0}^{1} t \psi_{1}(t) \mathrm{d} t\right]^{-1} .
$$

Now, note (82), and we see there is an $M>0$ such that
This, together with (H7), implies that

$$
\begin{array}{r}
f\left(s, \int_{0}^{s} \int_{0}^{\tau} y(r) \mathrm{d} r \mathrm{~d} \tau, \int_{0}^{s} y(\tau) \mathrm{d} \tau, y(s), y^{\prime}(s)\right) \leq H_{M}\left(y^{\prime}(s)\right), \\
\text { for } s \in[0,1] .
\end{array}
$$

Note that $y \in U$, and we obtain

$$
\begin{aligned}
-y^{\prime \prime}(t)= & -(A y)^{\prime \prime}(t)-\lambda \varphi_{2}^{\prime \prime}(t) \\
= & f\left(t, \int_{0}^{t} \int_{0}^{\tau} y(r) \mathrm{d} r \mathrm{~d} \tau, \int_{0}^{t} y(\tau) \mathrm{d} \tau, y(t), y^{\prime}(t)\right) \\
& +\lambda \leq H_{M}\left(y^{\prime}(t)\right)+\lambda .
\end{aligned}
$$


This implies that

$$
\frac{-y^{\prime \prime}(t) y^{\prime}(t)}{H_{M}\left(y^{\prime}(t)\right)+\lambda} \leq y^{\prime}(t),
$$

and then if we let $\varrho=y^{\prime}$, we have

$$
\begin{aligned}
\int_{0}^{\left\|y^{\prime}\right\|_{C} \frac{\varrho \mathrm{d} \varrho}{H_{M}(\varrho)+\lambda}} \leq \int_{0}^{1} \int_{0}^{1} y^{\prime}(t) \mathrm{d} t \\
=y(1)-y(0) \leq \frac{\kappa_{2} e_{2} \int_{0}^{1} \psi_{1}(t) \mathrm{d} t}{2 r\left(L_{a_{4}, b_{4}, c_{4}}^{*}\right)-2} \\
\cdot\left[\int_{0}^{1} t \psi_{1}(t) \mathrm{d} t\right]^{-1} .
\end{aligned}
$$

Therefore, combining this and (H7), there exists $\mathcal{N}^{*}>0$ such that

$$
\left\|y^{\prime}\right\|_{C} \leq \mathcal{N}^{*}
$$

Thus, $U$ is bounded (see (82) and (88)). Taking $R_{2}>\max \left\{\sup U, \rho_{2}\right\}$, we have

$$
y-A y \neq \lambda \varphi_{2}, \quad \text { for } y \in \partial B_{R_{2}} \cap P, \lambda \geq 0 .
$$

From Lemma 4, we have

$$
i\left(A, B_{R_{2}} \cap P, P\right)=0 .
$$

From (76) and (90), we have

$$
\begin{aligned}
i\left(A,\left(B_{R_{2}} \backslash \bar{B}_{\rho_{2}}\right) \cap P, P\right)= & i\left(A, B_{R_{2}} \cap P, P\right) \\
& -i\left(A, B_{\rho_{2}} \cap P, P\right)=-1,
\end{aligned}
$$

and hence $A$ has at least one fixed point in $\left(B_{R_{2}}, \bar{B}_{\rho_{2}}\right) \cap P$, i.e., (1) has at least one positive solution. This completes the proof.

\section{Data Availability}

No data were used to support this study.

\section{Conflicts of Interest}

The authors declare that they have no conflicts of interest.

\section{Acknowledgments}

This work was supported by the China Postdoctoral Science Foundation (grant no. 2019M652348), Technology Research Foundation of Chongqing Educational Committee (grant no. KJQN201900539), Natural Science Foundation of Chongqing Normal University (grant no. 16XYY24), and Shandong Natural Science Foundation (grant no. ZR2018MA011).

\section{References}

[1] Z. Bai, "Positive solutions of some nonlocal fourth-order boundary value problem," Applied Mathematics and Computation, vol. 215, no. 12, pp. 4191-4197, 2010.
[2] X. Hao, N. Xu, and L. Liu, "Existence and uniqueness of positive solutions for fourth-order $m$-point boundary value problems with two parameters," Rocky Mountain Journal of Mathematics, vol. 43, no. 4, pp. 1161-1180, 2013.

[3] Y. Wei, Q. Song, and Z. Bai, "Existence and iterative method for some fourth order nonlinear boundary value problems," Applied Mathematics Letters, vol. 87, pp. 101-107, 2019.

[4] Y. Pang and Z. Bai, "Upper and lower solution method for a fourth-order four-point boundary value problem on time scales," Applied Mathematics and Computation, vol. 215, no. 6, pp. 2243-2247, 2009.

[5] F. Zhu, L. Liu, and Y. Wu, "Positive solutions for systems of a nonlinear fourth-order singular semipositone boundary value problems," Applied Mathematics and Computation, vol. 216, no. 2, pp. 448-457, 2010.

[6] W. Fan, X. Hao, L. Liu, and Y. Wu, "Nontrivial solutions of singular fourth-order Sturm-Liouville boundary value problems with a sign-changing nonlinear term," Applied Mathematics and Computation, vol. 217, no. 15, pp. 6700-6708, 2011.

[7] K. Zhang, "Nontrivial solutions of fourth-order singular boundary value problems with sign-changing nonlinear terms," Topological Methods in Nonlinear Analysis, vol. 40, no. 1, pp. 53-70, 2012.

[8] Y. Zou, "On the existence of positive solutions for a fourthorder boundary value problem," Journal of Function Spaces, vol. 2017, p. 5, 2017.

[9] X. Zhang and Y. Cui, "Positive solutions for fourth-order singular $p$-Laplacian differential equations with integral boundary conditions," Boundary Value Problems, vol. 2010, p. 23, 2010 .

[10] Y. Cui and J. Sun, "Existence of multiple positive solutions for fourth-order boundary value problems in Banach spaces," Boundary Value Problems, vol. 2012, no. 1, p. 13, 2012.

[11] Y. Cui and Y. Zou, "Existence and uniqueness theorems for fourth-order singular boundary value problems," Computers \& Mathematics with Applications, vol. 58, no. 7, pp. 14491456, 2009.

[12] OA. Arqub, "An iterative method for solving fourth-order boundary value problems of mixed type integro-differential equations," Journal of Computational and Applied Mathematics, vol. 18, no. 5, pp. 857-874, 2015.

[13] A. Cabada and S. Tersian, "Multiplicity of solutions of a two point boundary value problem for a fourth-order equation," Applied Mathematics and Computation, vol. 219, no. 10, pp. 5261-5267, 2013.

[14] G. Bonanno and B. Di Bella, "Infinitely many solutions for a fourth-order elastic beam equation," Nonlinear Differential Equations and Applications NoDEA, vol. 18, no. 3, pp. 357368, 2011.

[15] Y. Li, "Existence of positive solutions for the cantilever beam equations with fully nonlinear terms," Nonlinear Analysis: Real World Applications, vol. 27, pp. 221-237, 2016.

[16] Z. Yang and J. Sun, "Positive solutions of a fourth-order boundary value problem involving derivatives of all orders," Communications on Pure and Applied Analysis, vol. 11, no. 5, pp. 1615-1628, 2012.

[17] K. Zhang, D. O’Regan, and Z. Fu, "Nontrivial solutions for boundary value problems of a fourth order difference equation with sign-changing nonlinearity," Advances in Difference Equations, vol. 2018, no. 1, p. 13, 2018.

[18] J. Liu and Z. Zhao, "On the nonhomogeneous fourth-order $p$ Laplacian generalized Sturm-Liouville nonlocal boundary 
value problems," Discrete Dynamics in Nature and Society, vol. 2012, p. 12, 2012.

[19] J. Jiang, L. Liu, and Y. Wu, "Positive solutions for p-Laplacian fourth-order differential system with integral boundary conditions," Discrete Dynamics in Nature and Society, vol. 2012, p. 19, 2012.

[20] Y. Cui and Y. Zou, "Existence and uniqueness of solutions for fourth-order boundary-value problems in Banach spaces, electron," Journal of Differential Equations, vol. 2009, no. 33, pp. 1-8, 2009.

[21] M. R. H. Tavani, "Existence results for fourth-order elastic beam equations on the real line," Dynamic Systems and Applications, vol. 27, no. 1, pp. 149-163, 2018.

[22] M. Tuz, "The existence of symmetric positive solutions of fourth-order elastic beam equations," Symmetry, vol. 11, no. 1, p. 121, 2019.

[23] N. Ghawadri, N. Senu, F. Adel Fawzi, F. Ismail, and Z. Ibrahim, "Diagonally implicit Runge-Kutta type method for directly solving special fourth-order ordinary differential equations with III-posed problem of a beam on elastic foundation," Algorithms, vol. 12, no. 1, p. 10, 2019.

[24] Y. Tian, S. Shang, and Q. Huo, "Antiperiodic solutions of fourth-order impulsive differential equation," Mathematical Methods in the Applied Sciences, vol. 41, no. 2, pp. 769-780, 2017.

[25] B. Azarnavid, K. Parand, and S. Abbasbandy, "An iterative kernel based method for fourth order nonlinear equation with nonlinear boundary condition," Communications in Nonlinear Science and Numerical Simulation, vol. 59, pp. 544-552, 2018.

[26] R. Jiang and C. Zhai, "Combined effects of concave and convex nonlinearities in nonperiodic fourth-order equations, electron," Electronic Journal of Qualitative Theory of Differential Equations, vol. 30, no. 30, pp. 1-14, 2018.

[27] Y. Zhang, J.-P. Sun, and J. Zhao, "Positive solutions for a fourth-order three-point BVP with sign-changing Green's function," Electronic Journal of Qualitative Theory of Differential Equations, vol. 5, no. 5, pp. 1-11, 2018.

[28] Y. Han, "A class of fourth-order parabolic equation with arbitrary initial energy," Nonlinear Analysis: Real World Applications, vol. 43, pp. 451-466, 2018.

[29] R. Behl, A. Cordero, S. S. Motsa, and J. R. Torregrosa, "Multiplicity anomalies of an optimal fourth-order class of iterative methods for solving nonlinear equations," Nonlinear Dynamics, vol. 91, no. 1, pp. 81-112, 2018.

[30] Z. Bai, Z. Du, and S. Zhang, "Iterative method for a class of fourth-order $p$-Laplacian beam equation," Journal of Applied Analysis and Computation, vol. 9, no. 4, pp. 1443-1453, 2019.

[31] Y. Li, Y. Ding, and E. Ibrahim, "Positive radial solutions for elliptic equations with nonlinear gradient terms on an exterior domain," Mediterranean Journal of Mathematics, vol. 15, no. 3, p. 83, 2018.

[32] Y. Li and Y. Li, "Positive solutions of a third-order boundary value problem with full nonlinearity," Mediterranean Journal of Mathematics, vol. 14, no. 3, p. 128, 2017.

[33] Y. Li, "Positive solutions for second-order boundary value problems with derivative terms," Mathematische Nachrichten, vol. 289, no. 16, pp. 2058-2068, 2016.

[34] J. Zhang, G. Zhang, and H. Li, "Positive solutions of secondorder problem with dependence on derivative in nonlinearity under Stieltjes integral boundary condition," Electronic Journal of Qualitative Theory of Differential Equations, vol. 4, no. 4, pp. 1-13, 2018.
[35] J. R. Cannon, "The solution of the heat equation subject to the specifcation of energy," Quarterly of Applied Mathematics, vol. 21, no. 2, pp. 155-160, 1963.

[36] N. I. Ionkin, "The solution of a certain boundary value problem of the theory of heat conduction with a nonclassical boundary condition," Journal of Differential Equations, vol. 13, no. 2, pp. 294-304, 1977.

[37] R. Y. Chegis, "Numerical solution of a heat conduction problem with an integral condition," Litovski Matematicheskiǔ Sbornik, vol. 24, no. 4, pp. 209-215, 1984.

[38] J. Wu, X. Zhang, L. Liu, Y. Wu, and Y. Cui, "The convergence analysis and error estimation for unique solution of a $p$ Laplacian fractional differential equation with singular decreasing nonlinearity," Boundary Value Problems, vol. 2018, no. 1, p. 15, 2018.

[39] X. Zhang, J. Wu, L. Liu, Y. Wu, and Y. Cui, "Convergence analysis of iterative scheme and error estimation of positive solution for a fractional differential equation," Mathematical Modelling and Analysis, vol. 23, no. 4, pp. 611-626, 2018.

[40] J. Webb, "Positive solutions of nonlinear differential equations with Riemann-Stieltjes boundary conditions," Electronic Journal of Qualitative Theory of Differential Equations, vol. 86, no. 86, pp. 1-13, 2016.

[41] B. Ahmad, Y. Alruwaily, A. Alsaedi, and S. K. Ntouyas, "Existence and stability results for a fractional order differential equation with non-conjugate Riemann-Stieltjes integro-multipoint boundary conditions," Mathematics, vol. 7, no. 3, p. 249, 2019.

[42] F. Wang, L. Liu, Y. Wu, and Y. Zou, "Iterative analysis of the unique positive solution for a class of singular nonlinear boundary value problems involving two types of fractional derivatives with $p$-Laplacian operator," Complexity, vol. 2019, p. 21, 2019.

[43] Q. Song and Z. Bai, "Positive solutions of fractional differential equations involving the Riemann-Stieltjes integral boundary condition," Advances in Difference Equations, vol. 2018, no. 1, p. 7, 2018.

[44] W. Ma and Y. Cui, "The eigenvalue problem for Caputo type fractional differential equation with Riemann-Stieltjes integral boundary conditions," Journal of Function Spaces, vol. 2018, p. 9, 2018.

[45] W. Ma, S. Meng, and Y. Cui, "Resonant integral boundary value problems for Caputo fractional differential equations," Mathematical Problems in Engineering, vol. 2018, p. 8, 2018.

[46] S. Meng and Y. Cui, "Multiplicity results to a conformable fractional differential equations involving integral boundary condition," Complexity, vol. 2019, p. 8, 2019.

[47] S. Meng and Y. Cui, "The uniqueness theorem of the solution for a class of differential systems with coupled integral boundary conditions," Discrete Dynamics in Nature and Society, vol. 2018, p. 7, 2018.

[48] X. Zhang, L. Liu, Y. Wu, and Y. Zou, "Existence and uniqueness of solutions for systems of fractional differential equations with Riemann-Stieltjes integral boundary condition," Advances in Difference Equations, vol. 2018, no. 1, p. 15, 2018.

[49] K. Deimling, Nonlinear Functional Analysis, Springer, Berlin, Germany, 1985.

[50] K. Chang and Q. Lin, The Lecture of Functional Analysis, The Press of Beijing University, Beijing, China, 2001.

[51] D. Guo and V. Lakshmikantham, Nonlinear Problems in Abstract Cones, Academic Press, Orlando, FL, USA, 1988. 


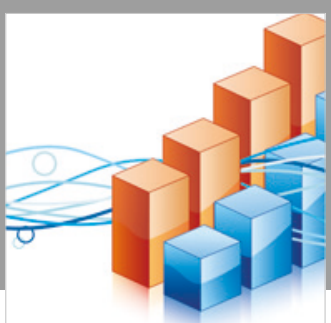

Advances in

Operations Research

\section{-n-m}
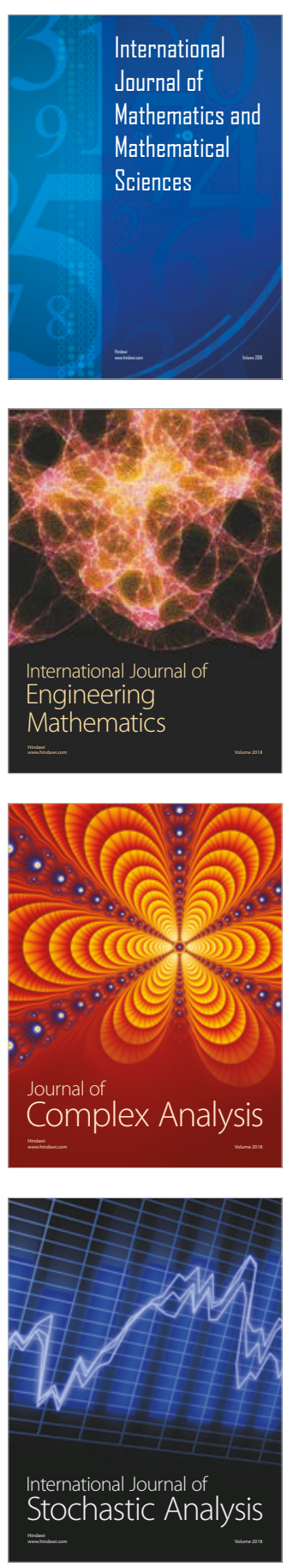
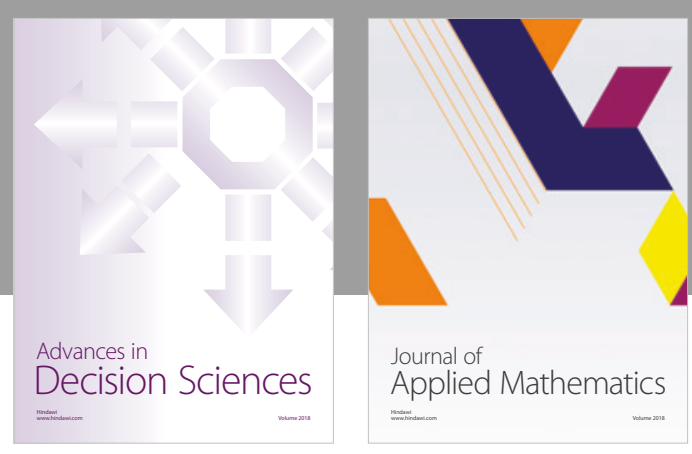

Journal of

Applied Mathematics
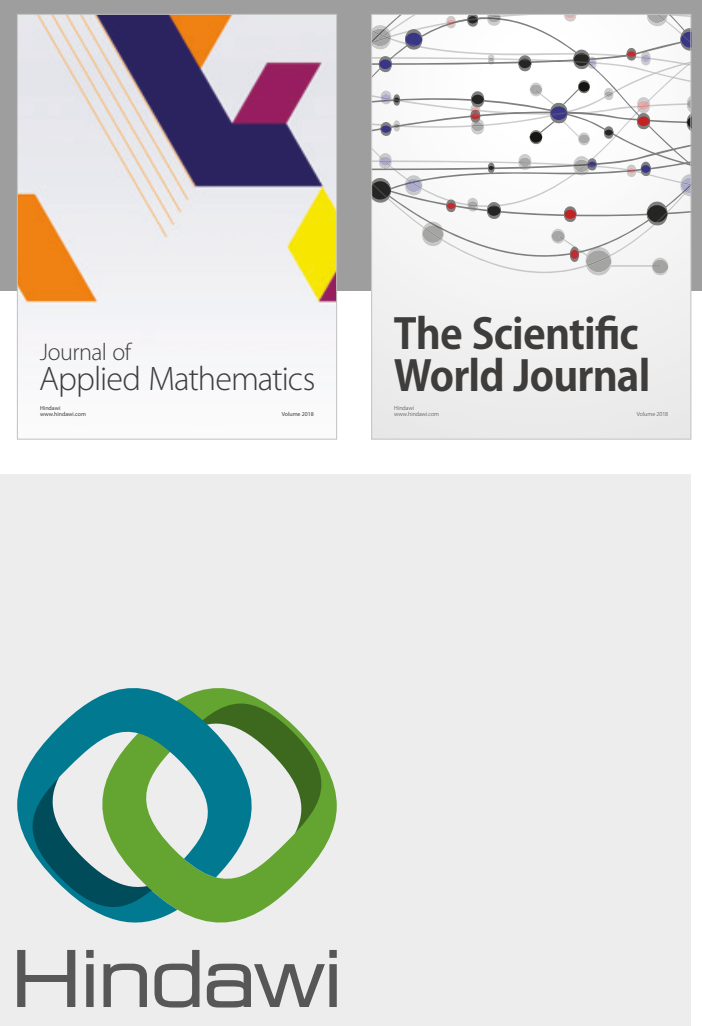

Submit your manuscripts at

www.hindawi.com

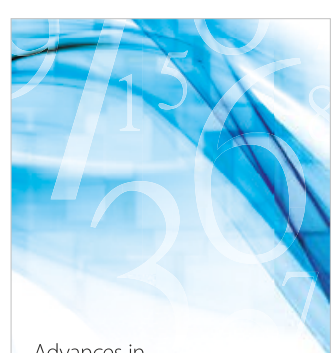

Advances in
Numerical Analysis
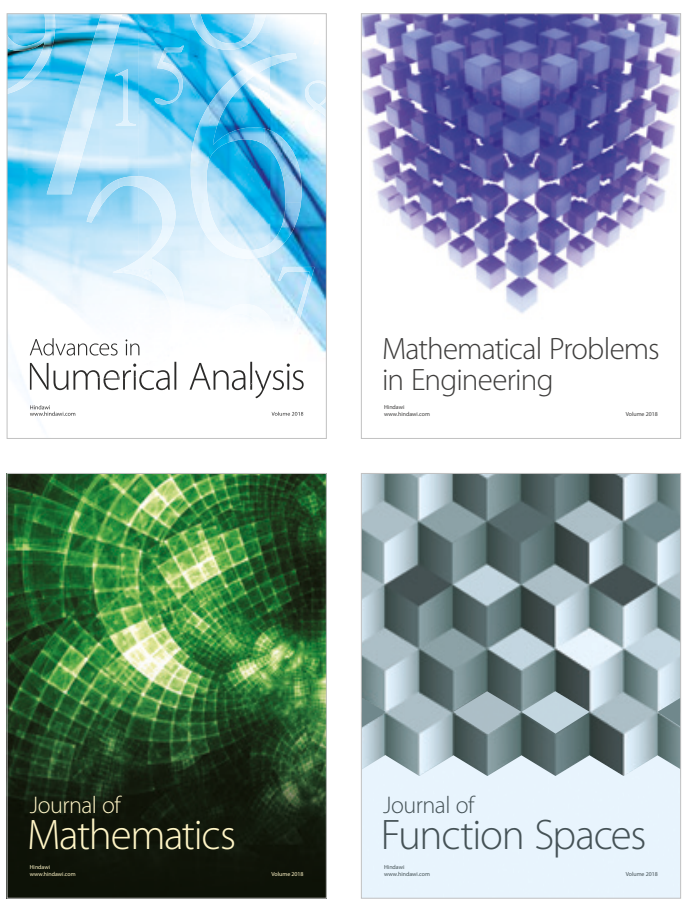

Mathematical Problems in Engineering

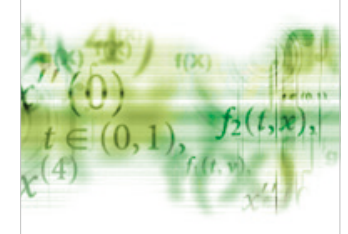

International Journal of

Differential Equations

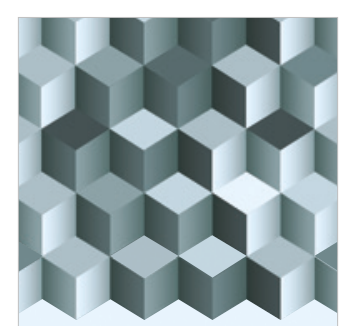

Journal of

Function Spaces

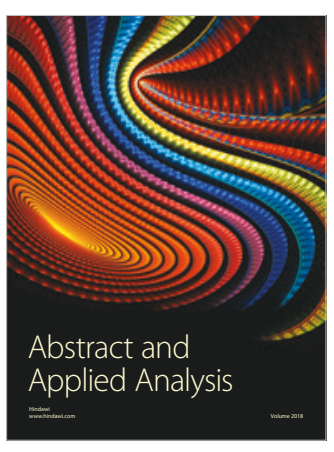

The Scientific

World Journal

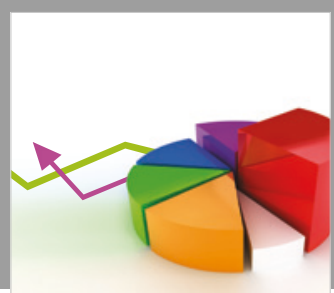

Journal of

Probability and Statistics
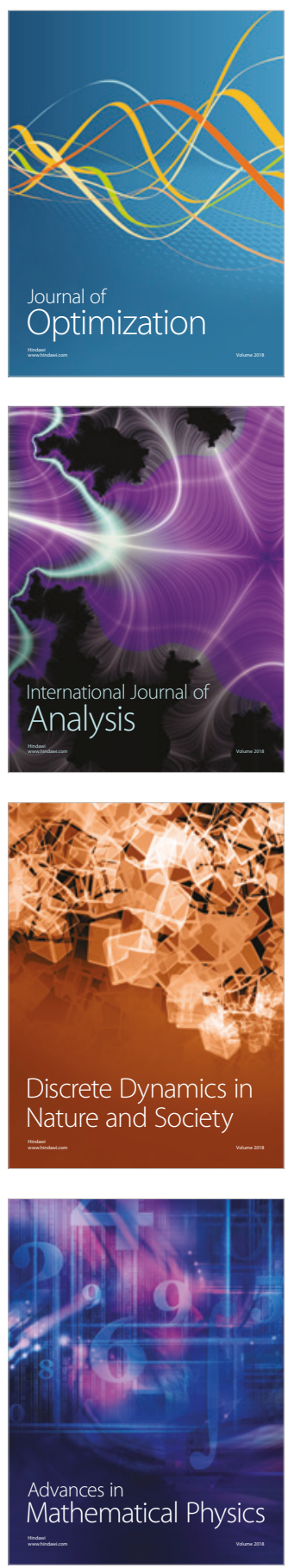